\title{
Damping parametric instabilities in future gravitational wave detectors by means of electrostatic actuators
}

\author{
John Miller ${ }^{\mathrm{a}, 1, *}$, Matthew Evans ${ }^{\mathrm{a}}$, Lisa Barsotti ${ }^{\mathrm{a}}$, Peter Fritschel ${ }^{\mathrm{a}}$, Myron MacInnis ${ }^{\mathrm{a}}$, \\ Richard Mittleman ${ }^{\mathrm{a}}$, Brett Shapiro ${ }^{\mathrm{a}}$, Jonathan Soto ${ }^{\mathrm{a}}$, Calum Torrie ${ }^{\mathrm{b}}$ \\ ${ }^{a}$ LIGO Laboratory, Massachusetts Institute of Technology, 185 Albany St, Cambridge, MA 02139, United \\ States of America \\ ${ }^{b}$ LIGO Laboratory, California Institute of Technology, 1200 E. California Blvd., Pasadena, CA 91125, \\ United States of America
}

\begin{abstract}
It has been suggested that the next generation of interferometric gravitational wave detectors may observe spontaneously excited parametric oscillatory instabilities. We present a method of actively suppressing any such instability through application of electrostatic forces to the interferometers' test masses. Using numerical methods we quantify the actuation force required to damp candidate instabilities and find that such forces are readily achievable. Our predictions are subsequently verified experimentally using prototype Advanced LIGO hardware, conclusively demonstrating the effectiveness of our approach.
\end{abstract}

Keywords: parametric instability, gravitational wave, interferometer, electrostatic drive PACS: 04.80.Nn, 95.55.Ym, 41.20.Cv

\section{Introduction}

The second generation of interferometric gravitational wave detectors [1, 2, 3] shall utilise test masses of high mechanical quality factor and operate with increased circulating power in their Fabry-Perot arm cavities. These changes are designed to reduce test mass thermal noise and quantum shot noise respectively (see e.g. [4, 5]). How-

\footnotetext{
*Corresponding author

Email address: john.miller@anu.edu.au (John Miller)

${ }^{1}$ Permanent address: Centre for Gravitational Physics, The Australian National University, Acton 0200, Australia. Tel: +61 26125 1012, Fax: +61 2 61250741.
}

Preprint submitted to Physics Letters A ever, current understanding suggests that these alterations will also make advanced detectors more susceptible to parametric instabilities - opto-acoustic interactions with the ability to excite mechanical eigenmodes of an interferometer's optics, compromising detector noise performance and control [6, 7, 8, 9].

A number of schemes are currently being investigated to mitigate this potential problem [10, 11]. Here we consider one such scheme employing electrostatic actuators to actively damp any instability in a frequency selective manner.

Using numerical methods we estimate the damping available from these actuators and

October 13, 2018 
find that it is sufficient to quash all theoretically dangerous modes. Our numerical results are subsequently verified experimentally using prototype Advanced LIGO hardware.

Although we focus our attention on the Advanced LIGO detectors our techniques are equally applicable to other long-baseline interferometers.

\subsection{Parametric instability}

The kilometre-scale Fabry-Perot arm cavities of second generation interferometric gravitational wave detectors are expected to store $\sim 1 \mathrm{MW}$ of optical power. The mirrors forming these cavities consist of multi-layer dielectric coatings deposited atop $40 \mathrm{~kg}$ cylinders $(34 \mathrm{~cm} \varnothing \times 20 \mathrm{~cm} \mathrm{tk}$. $)$ of fused silica. These cylinders are known as test masses and exhibit mechanical quality factors $\gtrsim 10^{7}$.

Parametric instabilities (PIs) result from the non-linear coupling of optical energy stored in an interferometer's arm cavities into mechanical energy stored in the internal mechanical modes of its test masses. This coupling is driven by ponderomotive radiation pressure forces and may be easily understood as a classical feedback effect [8].

Excitation, thermal or otherwise, of a test mass eigenmode initiates the process, scattering light from the fundamental cavity mode into sideband fields with frequency spacing equal to the mechanical mode frequency. The spatial profile of the test mass eigenmode is also imprinted onto these fields. The sidebands then experience the optical response of the interferometer, which is generally different for upper and lower sidebands, before arriving back to the excited optic together with the main cavity field. Radiation pressure couples energy from the scattered light into mechanical motion, thus closing the feedback loop. Based on overall loop phase, the mechanical mode may be suppressed or further excited, the latter case being potentially unstable. This process may be described by a single dimensionless quantity, the parametric gain $R_{m}$ [8],

$$
R_{m}=\frac{4 \pi Q_{m} P_{\text {circ }}}{M \omega_{m}^{2} c \lambda_{0}} \sum_{n=0}^{\infty} \Re\left[G_{n}\right] B_{m, n}^{2} .
$$

Here $Q_{m}$ is the quality factor of test mass mode $m, \omega_{m}$ is its angular resonant frequency, $M$ is the mass of the test mass, $P_{\text {circ }}$ is the circulating optical power in the main cavity mode and $\lambda_{0}$ is its wavelength. $B_{m, n}$ is the geometric overlap of mechanical mode $m$ with optical eigenmode $n$. $\Re\left[G_{n}\right]$ is the real part of the complex transfer coefficient from field $n$ leaving an optic's surface to a field incident on and then reflected from the same surface; for details of its calculation see [8] and references therein.

Modelling the system as a feedback loop, $R_{m}$ is nothing other than the real part of the open loop gain. Thus instability occurs for $R_{m}>1$. The parametric gain has been evaluated for an Advanced LIGO configuration (see table 1), including stable recycling cavities and diffraction loss, using the "worst case" methods of Evans et al. 8]. Results, shown in figure 1, reveal a number of potentially unstable modes.

As our analysis suggests, parametric instabilities may prove troublesome in second generation interferometers. Hence, efforts are ongoing to evaluate methods of ameliorating them. Schemes currently under study fall into two broad categories: those which modify $G_{n}$ [12, 13] and those which modify $Q_{m}$ [10, 14]. In this work we investigate the possibility of modifying the $Q$ of 
Table 1: Interferometer parameters used in the theoretical evaluation of parametric gain. These values correspond to those of Advanced LIGO.

\begin{tabular}{llc}
\hline & Quantity & Value \\
\hline \multirow{3}{*}{ Lengths } & Arm cavity & $3994.5 \mathrm{~m}$ \\
& Power recycling cavity & $57.175 \mathrm{~m}$ \\
& Signal recycling cavity & $55.475 \mathrm{~m}$ \\
\hline \multirow{3}{*}{ Gouy phases } & Arm cavity & $156^{\circ}$ \\
& Power recycling cavity & $25^{\circ}$ \\
& Signal recycling cavity & $20^{\circ}$ \\
\hline \multirow{4}{*}{ Optical } & Circulating arm power & $1 \mathrm{MW}$ \\
properties & Input mirror power transmittance & 0.014 \\
& End mirror power transmittance & $10^{-5}$ \\
& Power recycling mirror power transmittance & 0.03 \\
& Signal recycling mirror power transmittance & 0.2 \\
Mechanical & Beam splitter power transmittance & 0.5 \\
properties & Laser wavelength & $1064 \mathrm{~nm}$ \\
\hline
\end{tabular}

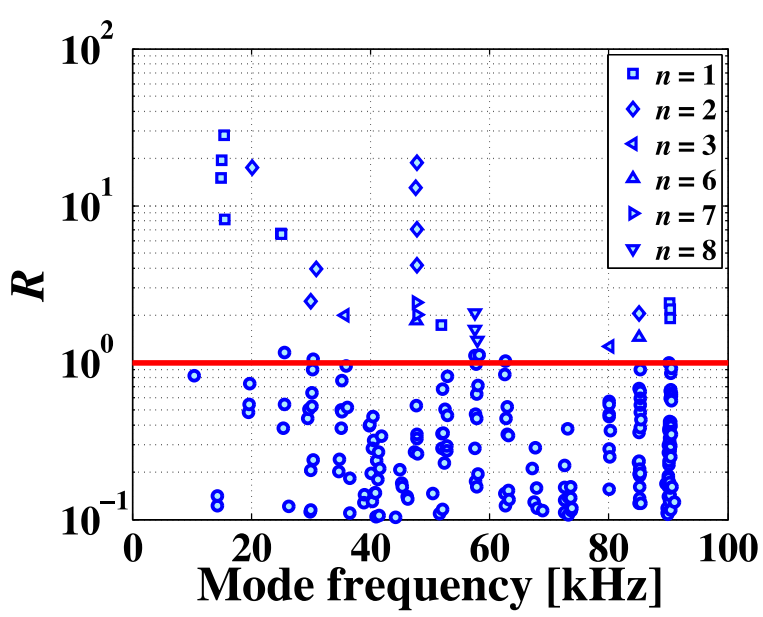

Figure 1: "Worst case" $R_{m}$ values for an interferometer configuration representative of Advanced LIGO. In our evaluation we randomly sweep over realistic model parameters, setting an upper limit on $R$ for each mode as the lowest value greater than $99.5 \%$ of the results. Diffraction losses are included via the clipping approximation. We find 212 modes with $R>0.1$ and 32 modes with $R>1$. For selected instabilities, the order of the optical mode most strongly excited is given in the legend. test mass mechanical modes using an electrostatic actuator.

\subsection{Electrostatic drive}

The majority of currently operating gravitational wave detectors utilise coil-magnet actuators to control the position and angular orientation of their suspended optics. The permanent magnets attached to the interferometer test masses are known to introduce noise through a number of mechanisms [15, 16, 17]. In order to eliminate these problems, Advanced LIGO uses Electrostatic Drives (ESDs) for the control of its most sensitive optics. Similar ESDs have been used successfully in the GEO 600 interferometer for some time [18].

For concreteness and in order to facilitate verification by experiment, we limit our discussion to the particular ESD shown in figure 2. This ESD, an Advanced LIGO pro- 


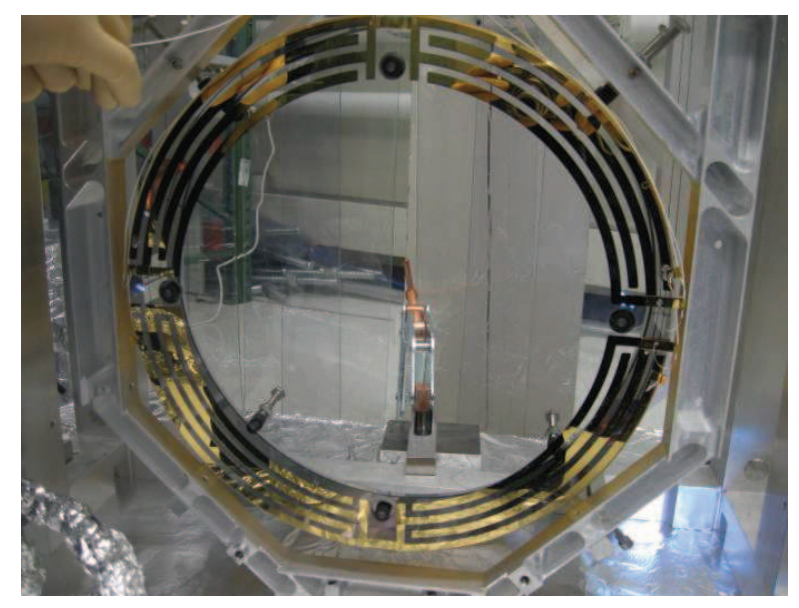

Figure 2: Advanced LIGO noise prototype reaction mass and ESD. This pattern is used for all numerical and experimental work discussed herein.

totype considered for control of the input test masses, consists of a symmetric pattern of four electrode pairs. These electrodes are deposited in gold on the surface of a fused silica reaction mass. The reaction mass is positioned immediately behind the test mass with the ESD electrodes facing its rear surface, so that the electrostatic force is applied between the two masses. The nominal inter-mass spacing is $5 \mathrm{~mm}$. To attenuate seismic disturbances both the reaction mass and test mass are suspended from isolated platforms by quadruple pendulums (see figure 3).

A potential difference between the ESD's electrode pairs gives rise to fringing electric fields which attract the dielectric test mass. This attractive force is proportional to the square of the potential difference and may be characterised by

$$
F_{\mathrm{ESD}}=\alpha(\Delta V)^{2},
$$

where $\Delta V$ is the potential difference between the electrode pairs and $\alpha$ is a constant of proportionality dependent on the separation between the test mass and reaction mass, the material properties of the

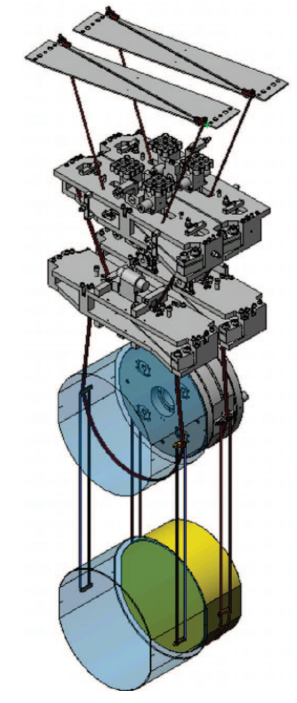

Figure 3: Engineering rendering of Advanced LIGO quadruple suspension system. The upper pendulum stages will continue to use coil-magnet actuators with only the critical final stage meriting an ESD. The ESD pattern is deposited onto the surface of the reaction mass (highlighted in yellow) adjacent to the test mass. Figure 2 shows a prototype reaction mass in more detail.

two optics and the specific geometry of the ESD pattern. With four separate electrode pairs, both angular orientation and longitudinal position can be controlled.

\section{Modelling}

\subsection{Required force}

In order for the ESD to effectively damp any parametric instability it must have a discernible effect on the $Q$ of test mass mechanical modes. Using arguments outlined fully in Appendix A we find that, for a thermally excited mode, the magnitude of force demanded from the ESD to reduce the parametric gain from $R_{m}$ to $R_{\mathrm{eff}, m}$ is

$$
F_{\mathrm{ESD}, m}=\sqrt{\mu_{m} k_{B} T} \frac{\omega_{0, m}}{b_{m}}\left(\frac{R_{m}-R_{\mathrm{eff}, m}}{Q_{m} R_{\mathrm{eff}, m}}\right) .
$$


Here $\omega_{0, m}$ is the angular eigenfrequency of mode $m, \mu_{m}$ is its modal mass and $Q_{m}$ its mechanical quality factor. $T$ is the ambient temperature, taken to be $300 \mathrm{~K}$, and $k_{B}$ is Boltzmann's constant. The parameter $b_{m}$, defined via A.2 , represents the fraction of applied ESD force which is coupled into mechanical mode $m$. Given the difficulties involved in evaluating this parameter analytically, a numerical approach was employed.

\subsection{Evaluating $b_{m}$}

In order to calculate $b_{m}$ one requires knowledge of both the test mass eigenmodes and the spatial distribution of ESD forces.

Mode shapes and frequencies of an Advanced LIGO test mass, including the flat regions on the barrel of the mass ${ }^{2}$ but not the 'ears' which link the mass to its suspension fibres, were found using commercial finite element software [20, 21]. This analysis produced several thousand eigenmodes with frequencies in the $10-90 \mathrm{kHz}$ band. We limit ourselves to this region as higher frequency modes typically couple to high order optical modes which suffer from significant diffraction losses and thus yield low parametric gain. In our investigation only modes with appreciable parametric gain $\left(R_{m}>0.1\right)$ were considered.

As a consequence of the complex electrode geometry and non-uniform electric fields involved, the calculation of the force produced by the ESD is also well suited to finite element analysis. An appropriate model was constructed and solved for the electrostatic potential. Selected model parameters are presented in table 2 .

With a view to finding the ESD pressure distribution $p_{\mathrm{ESD}}$ across the transverse

\footnotetext{
${ }^{2}$ The inclusion of flat regions splits modes without axial symmetry into doublet pairs [19].
}

Table 2: Parameters used in modelling the performance of the electrostatic drive.

\begin{tabular}{lc}
\hline Quantity & Value \\
\hline Test mass radius & $17 \mathrm{~cm}$ \\
Test mass thickness & $20 \mathrm{~cm}$ \\
Reaction mass radius & $17 \mathrm{~cm}$ \\
Reaction mass thickness & $13 \mathrm{~cm}$ \\
Inter-mass spacing & $5 \mathrm{~mm}$ \\
\hline Relative permittivity of masses & 3.75 \\
Inner radius of electrode pattern & $13.3 \mathrm{~cm}$ \\
Outer radius of electrode pattern & $16.8 \mathrm{~cm}$ \\
Electrode thickness & $5 \mathrm{~mm}$ \\
Electrode spacing & $5 \mathrm{~mm}$ \\
\hline
\end{tabular}

dimensions of the test mass, the Maxwell stress tensor $(\mathrm{MST}) \Upsilon_{i j}$ was invoked. For our electrostatic simulation

$$
\Upsilon_{i j}=E_{i} D_{j}-\frac{1}{2} \delta_{i j} \sum_{k=1}^{3} E_{k} D_{k}
$$

where $E_{i}$ and $D_{i}$ are the $i^{\text {th }}$ Cartesian components of electric and displacement fields and $\delta_{i j}$ is the Kronecker delta. Integrating the MST over discrete closed volumes within the test mass, the pressure was obtained as a function of position (see figure 4). For further detail regarding this calculation see [22].

With mode shapes and pressure distribution available, the theoretical $b_{m}$ force coupling coefficients were found (via A.2 ) and used to evaluate the force required to damp parametric instabilities using an electrostatic drive.

\subsection{Modelling results}

A critical parameter for lock acquisition and interferometer control is the peak force available from the ESD. Integrating 


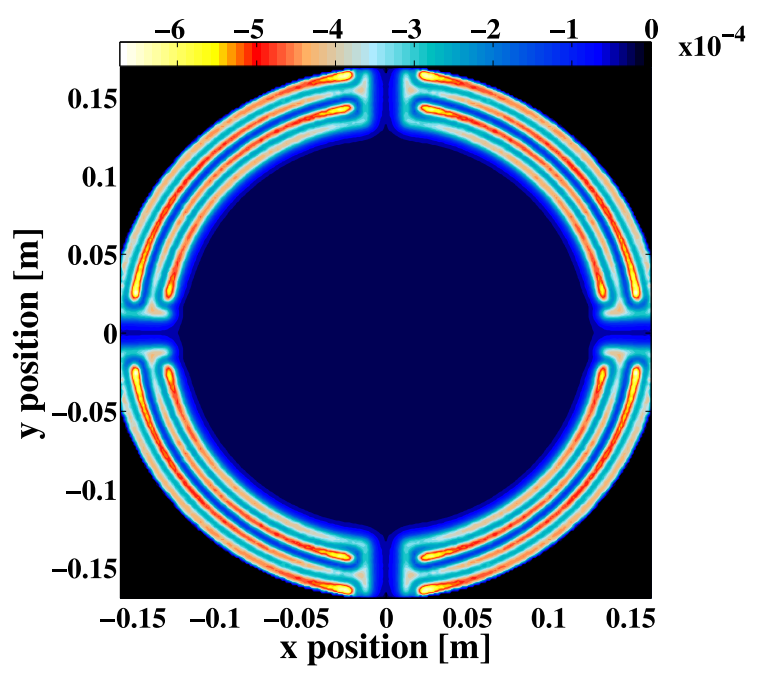

Figure 4: ESD pressure distribution in $\mathrm{Pa}$ as a function of transverse position across the test mass. Forces are uniformly attractive (negative) and most significant in the vicinity of the electrode pattern. $\Delta V=200 \mathrm{~V}$ is assumed.

$p_{\mathrm{ESD}}$ over its domain ${ }^{3}$ we find the maximum available force to be $\sim 190 \mu \mathrm{N}$ for $\Delta V=800 \mathrm{~V}$, a reasonable value for a wide bandwidth, low-noise voltage amplifier and one considered for Advanced LIGO. This force corresponds to $\alpha=2.9 \times 10^{-10} \mathrm{~N} / \mathrm{V}^{2}$.

Figure 5 shows the fraction of this total force which must be drawn upon to reduce the effective parametric gain of each mode to 0.1 or less, a tolerable value for long-baseline interferometers. In all cases we see that successful damping is theoretically achievable using just a few percent of the available force.

Due to diffraction loss, optical gain is low for higher order transverse modes. This suggests that lower order optical modes, excited by low order test mass eigenmodes, are most likely to give rise to PIs. These low order mechanical modes exhibit low order symmetries, implying that their coupling to

\footnotetext{
${ }^{3}$ Or equivalently integrating the MST over the test mass boundary.
}

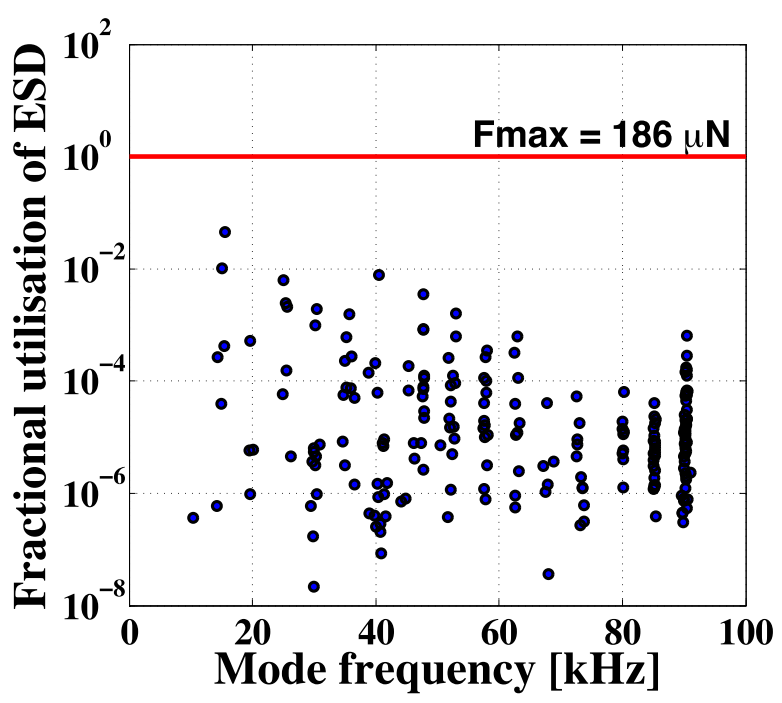

Figure 5: Fraction of total force available from all four ESD quadrants required to reduce the parametric gain to 0.1 . Maximum utilisation is $4.6 \%$. Available force evaluated for $\Delta V=800 \mathrm{~V}$.

the four-fold symmetric ESD pattern will be poor and that they will thus require large damping forces (see figure 6).

To combat this effect asymmetric ESD patterns were explored. It was quickly recognised that large benefits were available by operating with just a single ESD quadrant. Whilst median values of the damping force are comparable in both cases, the maximum utilisation of available force is reduced by more than an order of magnitude. This leads us to recommend single quadrant operation as the baseline mode for our scheme.

Operating in this way will also allow ESD damping to remain effective even if the test mass-reaction mass separation is increased from the nominal value of $5 \mathrm{~mm}$

\footnotetext{
${ }^{4}$ The test mass-reaction mass separation at the ITMs may be increased by a factor of $\sim 4$ to reduce gas damping noise 23. Although the available force will fall by factor of $\sim 40$ effective damping is still realisable.
} 


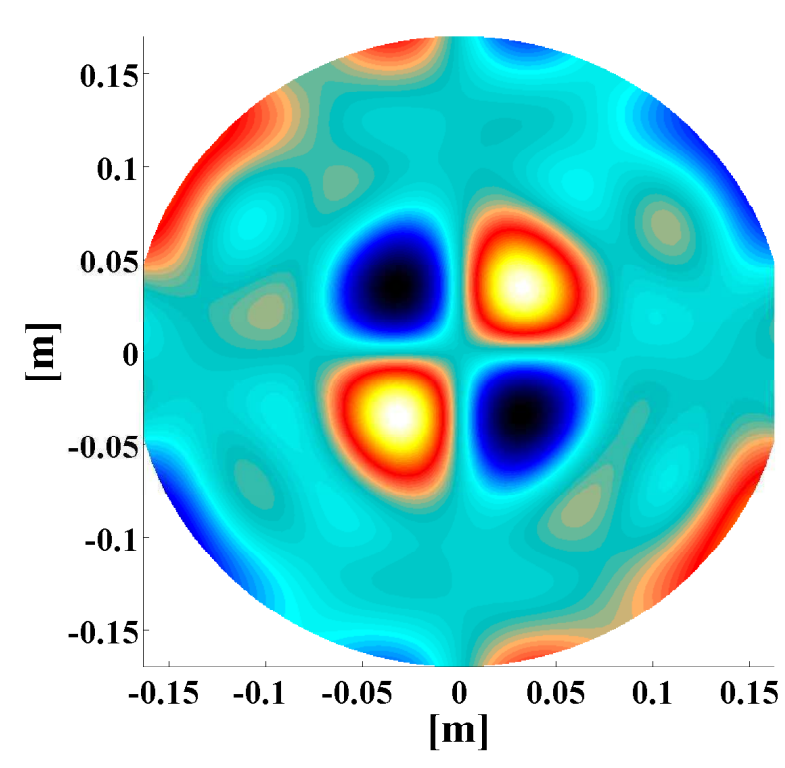

Figure 6: Mechanical modes with low order symmetries could prove difficult to damp using the fourfold symmetric ESD. This problem can be mitigated by introducing small asymmetries to the ESD pattern, by operating with just a single ESD quadrant or by recruiting all four ESD quadrants with mode dependent phasing.

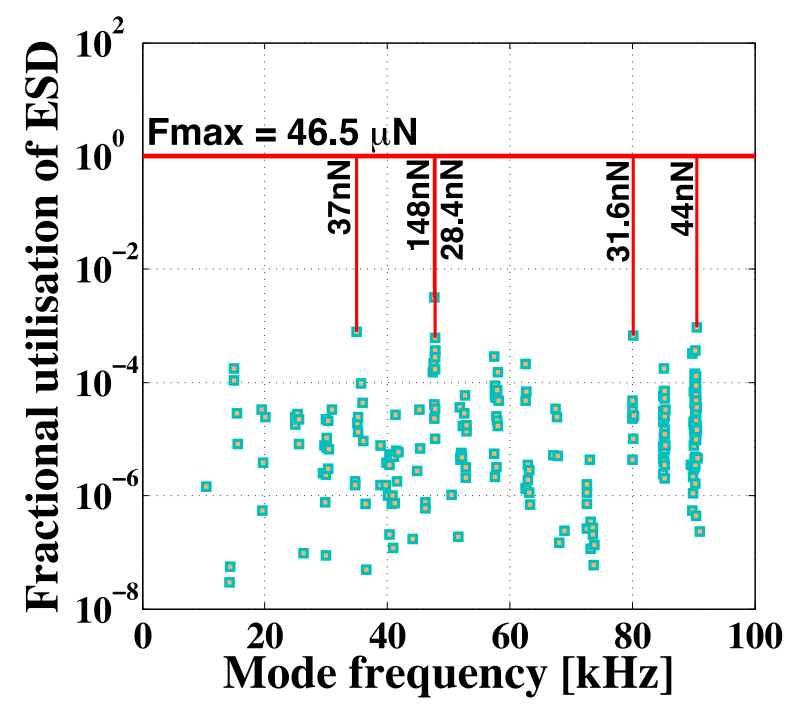

Figure 7: Fractional utilisation of available force to realise $R=0.1$ in single quadrant operation. Maximum utilisation is $0.3 \%$. The absolute force required to damp each of the five most demanding modes is indicated. Again, $\Delta V=800 \mathrm{~V}$ is assumed.
The fraction of available force utilised in single quadrant operation is shown in figure 7. Should additional actuation be required, the various quadrants can be recruited simultaneously with mode dependent phasing.

\section{Experimental verification}

\subsection{Apparatus}

In order to confirm the predictions of our numerical model an experimental investigation was carried out. All measurements were made by studying induced length fluctuations of an independently suspended, digitally controlled, 16 m Fabry-Perot optical resonator.

Our work utilised several Advanced LIGO prototype systems. Most notably, the cavity end mirror was a prototype $40 \mathrm{~kg} \mathrm{Ad}-$ vanced LIGO test mass, suspended by the quadruple pendulum system shown in figure 3 and controlled by the ESD shown in figure 2. The cavity input mirror was not used for prototyping test mass assemblies and thus had only a triple suspension and no reaction mass.

The resonator was driven by a sub-W Nd:YAG NPRO, frequency stabilised to a rigid reference cavity. Control of the suspended cavity was achieved using standard techniques [24] with control signals being applied to the coil-magnet actuators of the triple suspension. The unity gain frequency of the suspended cavity servo was set to 100 $\mathrm{Hz}$.

With this apparatus we were able to measure $\alpha$ and investigate our theoretically predicted couplings, $b_{m}$.

\subsection{The ESD actuation coefficient $\alpha$}

Our numerical prediction for $\alpha$ was tested experimentally by driving the cavity length 
at low frequencies $(10-100 \mathrm{~Hz})$ using all four quadrants of the ESD and measuring the response in the cavity error signal. To maximise available force, the potential of all electrodes was set to the largest possible value, with the two electrodes in each pair having opposite polarity.

For excitation frequencies above the highest pendulum resonance and below the first internal mode of the test mass we may write the peak-to-peak ESD force as

$$
F_{\mathrm{pp}}=M \omega_{\mathrm{exc}}^{2} x_{\mathrm{pp}},
$$

where $M$ is again the mass of the test mass, $\omega_{\text {exc }}$ is our excitation frequency in $\mathrm{rads}^{-1}$ and $x_{\mathrm{pp}}$ is the peak-to-peak test mass displacement along the cavity direction.

For known excitation waveforms, the peak-to-peak differential voltage $\Delta V_{\mathrm{pp}}$ applied to the ESD is also known. Thus from (2) we have

$$
\alpha=\frac{F_{\mathrm{pp}}}{\Delta V_{\mathrm{pp}}^{2}} .
$$

Measurements of $x_{\mathrm{pp}}$ were made as a function of excitation amplitude and frequency; $\alpha$ was found to have an mean value of $2.95 \pm 0.08 \times 10^{-10} \mathrm{~N} / \mathrm{V}^{2}$ (see figure 8). Accord with the predicted value of $2.91 \times 10^{-10} \mathrm{~N} / \mathrm{V}^{2}$ is excellent.

\subsection{The ESD-eigenmode coupling coeffi- cients $b_{m}$}

The force coupling between the ESD and test mass mechanical modes is of key importance in accurately quantifying our capacity to damp instabilities. In order to validate the theoretically calculated values, a series of measurements was made.

A simple feedback loop was implemented to excite the mechanical mode of interest into steady state oscillation. Breaking the loop allowed ringdown measurements to be made.

Knowing the steady state modal amplitude achieved for a given drive waveform and having measured the $Q$ via ringdowns, the ESD-mechanical mode overlap was found by modelling the system as a simple oscillator with angular resonant frequency $\omega_{0}$. At equilibrium, we have for each mode

$$
b_{m}=\frac{\omega_{0, m}^{2} \mu_{m} A_{m}}{Q_{m} F_{\mathrm{ESD}, m}},
$$

where $\mu_{m}$ is the modal mass of mode $m$.

The amplitude of $F_{\mathrm{ESD}, m}$ is calculated as

$$
F_{\mathrm{ESD}, m}=\frac{1}{2} \alpha\left[V_{\mathrm{exc}, m}-V_{\mathrm{bias}}\right]^{2},
$$

where $V_{\text {exc }}$ is the analogue ${ }^{5}$ excitation voltage applied to the inner ESD electrode and $V_{\text {bias }}$ the constant bias voltage applied to the outer electrode.

The modal amplitude $A_{m}$ is determined from the cavity error signal via $A_{m}=x_{m} / c_{m}$, where $x_{m}$ is the measured test mass displacement along the cavity direction and $c_{m}$ is a geometric overlap factor which accounts for the fraction of mechanical mode amplitude which is sensed by the cavity []$^{6}$ This parameter is calculated as

$$
c_{m}=\left|\iint_{\mathcal{S}} I_{00}\left(\vec{u}_{m} \cdot \hat{z}\right) \mathrm{d} \mathcal{S}\right| .
$$

Here $\vec{u}_{m}$ has the same normalisation as before (see (A.3) ) and $I_{00}$, the intensity profile

\footnotetext{
${ }^{5}$ The internal modes of the test mass lay beyond the Nyquist frequency $(\sim 8 \mathrm{kHz})$ of our digital system.

${ }^{6}$ For example, if the cavity beam is incident on the mirror near a node $c$ will be smaller than if the beam were incident near an anti-node.
} 


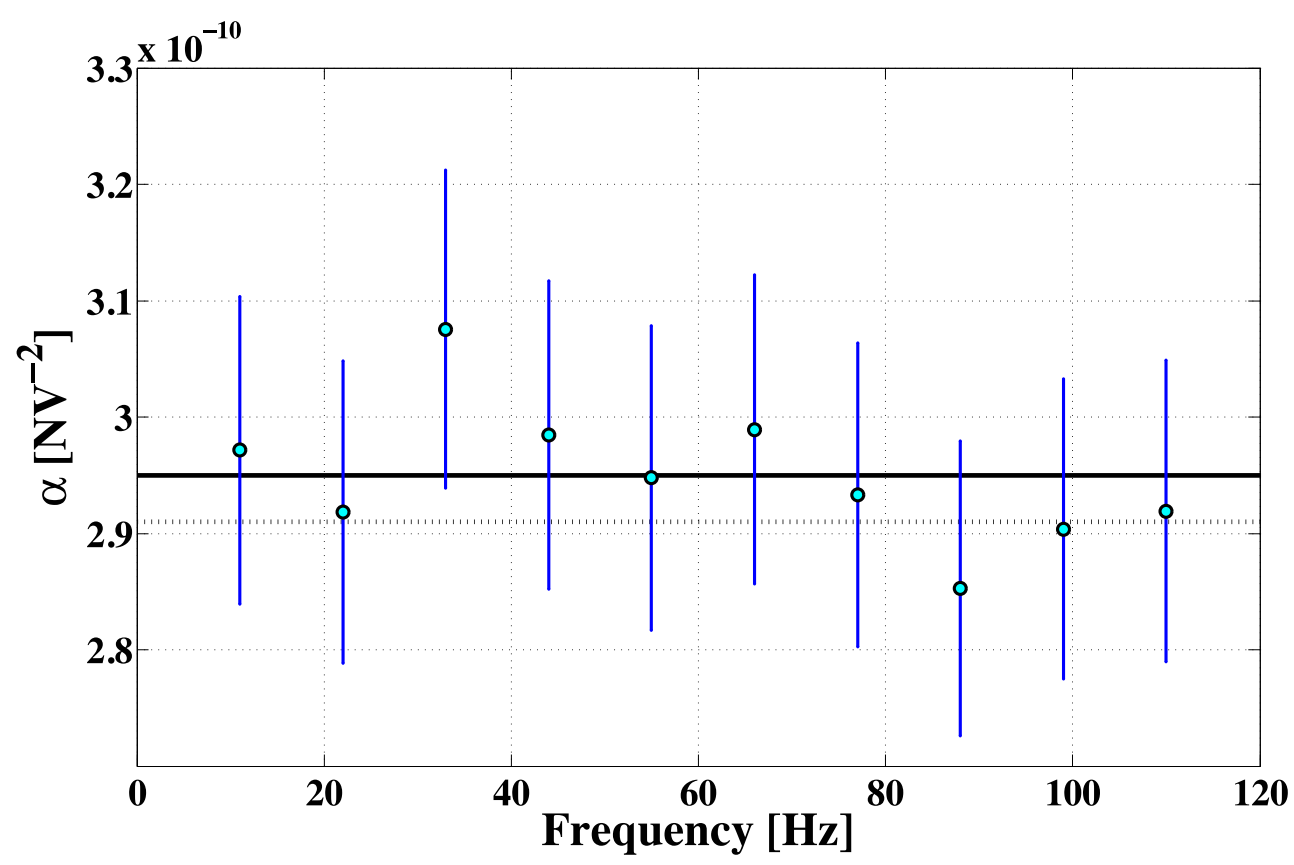

Figure 8: ESD actuation coefficient measurement. The solid line indicates the mean value of $2.95 \times 10^{-10} \mathrm{~N} / \mathrm{V}^{2}$; the dashed line shows the theoretically predicted value of $2.91 \times 10^{-10} \mathrm{~N} / \mathrm{V}^{2}$.

of the cavity's principal optical mode, has an integrated power of $1 \mathrm{~W}$.

In evaluating $c_{m}$ we make two assumptions. Firstly, that we know the shape of the mode which has been excited and secondly, that the cavity optical mode is centred on the test mass.

Table 3: Experimental mode parameters.

\begin{tabular}{ccc}
\hline $\begin{array}{c}\text { f measured } \\
\mathrm{kHz}\end{array}$ & $\begin{array}{c}\text { f predicted } \\
\mathrm{kHz}\end{array}$ & $Q$ \\
\hline 8.149 & 8.1439 & $412,170 \pm 1003$ \\
10.4115 & 10.397 & $604,158 \pm 4728$ \\
12.9705 & 12.959 & $508,666 \pm 2473$ \\
15.0405 & 15.0439 & $409,504 \pm 4139$ \\
\hline
\end{tabular}

The close agreement between the mode frequencies predicted by finite element modelling and those observed experimentally (see table 3) gives us confidence that the correct mode shapes were considered.
To mitigate any errors introduced by deviations from perfect centring we limited our analysis to 'drumhead' type modes with smoothly varying displacements near their centres. The four modes considered are shown in figure 9 .

Experimentally measured $Q$ values, listed in table 3, were found to be significantly lower than the value of $10^{7}$ we assume in our theoretical calculations. Note, however, that these $Q \mathrm{~s}$ were measured with a metal wire suspension; Advanced LIGO will adopt a quasi-monolithic fused silica design [25]. The test mass discussed herein has recently been suspended from silica fibres. Initial measurements confirm that $Q$ s of order $10^{7}$ will be achievable in second generation interferometers.

Using the apposite experimentally measured information, (5) was evaluated to yield $b_{m}$. Comparing our results to theoretical predictions, agreement was found to 


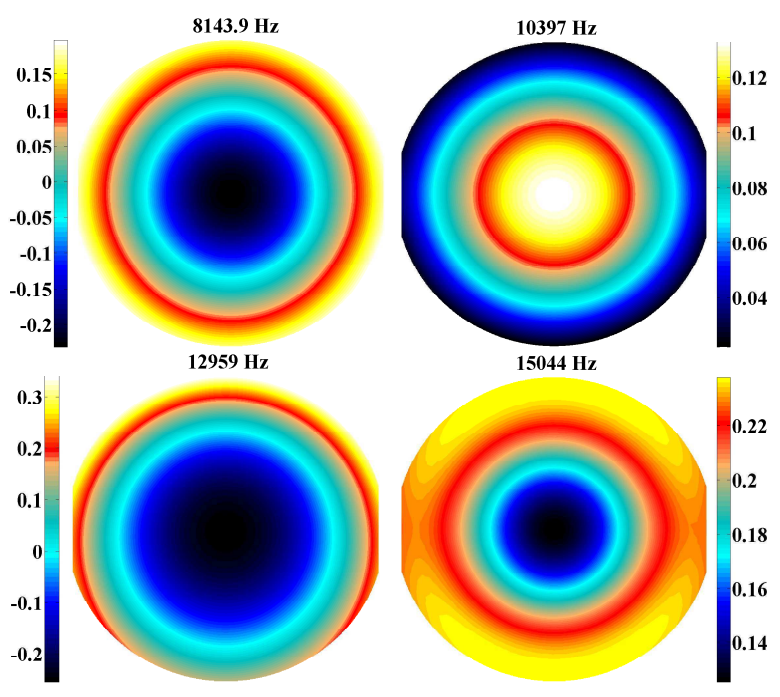

Figure 9: Modelled face displacements, $\vec{u}_{m} \cdot \hat{z}$, of the experimentally studied modes. The asymmetry in the $13 \mathrm{kHz}$ mode is due to the wedge angle of the test mass.

be better than $20 \%$. The ratio of experimental to theoretical overlap coefficients is plotted in figure 10 .

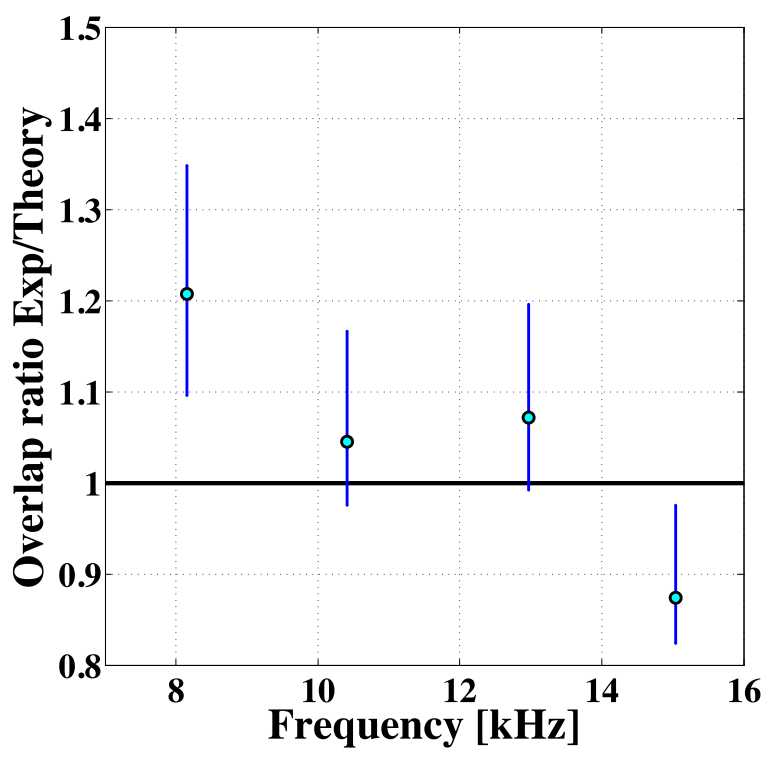

Figure 10: Ratio of experimental to theoretical overlap coefficients $b_{m}$ for the test mass modes of figure 9 .

\section{Summary and conclusions}

By numerical methods we have obtained the eigenmodes of a prototype Advanced LIGO test mass and the pressure distribution available from the electrostatic actuator used in its control. Having experimentally verified both quantities, this information was used to predict the force required to damp parametric instabilities in Advanced LIGO. We find that such forces are readily available. This result clearly demonstrates that electrostatic actuators do indeed represent a viable means of damping parametric instabilities.

In contrast with other techniques [10, 14], our damping scheme will not introduce any additional thermal noise. Further, due to the frequency selective nature of our approach, we also anticipate that any sensing noise injected by the ESD will be negligible. Unless a test mass has an appropriately placed eigenmode it will not exhibit any appreciable response to our damping forces $7^{7}$ What little coupling there is will be evident outside of the gravitational wave detection band.

There will of course be practical challenges to using electrostatic damping to control parametric instabilities. For example, it is possible for a number of modes to be above the instability threshold simultaneously. Although a feature of ESD damping is its ability to target each mode individually, this would also necessitate implementing a discrete control loop for each mode, increasing complexity as the number of problematic modes increased.

Each instability must also be sensed through the presence of the higher order optical modes which it excites. Extraction of

\footnotetext{
${ }^{7}$ This fact also allows us to apply damping forces to all test masses with impunity.
} 
these signals is complicated by the coupled resonant cavities found in advanced interferometers.

If PIs really do begin to threaten the robust operation of second generation interferometers there will likely be no single solution. It is probable that a combination of approaches will be employed, perhaps based around passive damping with an active scheme like ours targeted to particularly troublesome modes. Electrostatic actuators have proven themselves to be effective in suppressing test mass $Q \mathrm{~s}$ and are therefore capable of playing a major role in any such mitigation scheme.

\section{Appendix A. Force required to damp mechanical modes}

Considering each acoustical mode of the test mass as a damped oscillator with resonant frequency $\omega_{0, m} \operatorname{rads}^{-1}$ and modal mass $\mu_{m}$ we have

$$
\mu_{m} \ddot{x}_{m}+\frac{\omega_{0, m} \mu_{m}}{Q_{m}} \dot{x}_{m}+k_{m} x_{m}-F_{\text {app }, m}=0,
$$

where $F_{\mathrm{app}, m}$ is the force applied by the ESD. Assuming viscous damping

$$
F_{\text {app }, m}=-K_{m} \dot{x}_{m},
$$

we write

$$
\mu_{m} \ddot{x}_{m}+\left[\frac{\omega_{0, m} \mu_{m}}{Q_{\mathrm{eff}, m}}\right] \dot{x}_{m}+k_{m} x_{m}=0,
$$

in doing so defining an effective $Q$,

$$
Q_{\mathrm{eff}, m}=\left(\frac{1}{Q_{m}}+\frac{K_{m}}{\mu_{m} \omega_{0, m}}\right)^{-1},
$$

whence

$$
K_{m}=\mu_{m} \omega_{0, m}\left(\frac{1}{Q_{\mathrm{eff}, m}}-\frac{1}{Q_{m}}\right) .
$$

Therefore, asserting that $\dot{x}_{m}=j \omega_{0, m} x_{m}$,

$$
F_{\mathrm{app}, m}=j \mu_{m} \omega_{0, m}^{2} x_{m}\left(\frac{1}{Q_{m}}-\frac{1}{Q_{\mathrm{eff}, m}}\right) .
$$

For thermal excitations, $x_{m}=\left(k_{B} T / \mu_{m} \omega_{0, m}^{2}\right)^{1 / 2}$, we have

$$
F_{\text {app }, m}=j \omega_{0, m} \sqrt{\mu_{m} k_{B} T}\left(\frac{1}{Q_{m}}-\frac{1}{Q_{\mathrm{eff}, m}}\right) .
$$

We now introduce an additional parameter $b_{m}$, defined through $F_{\text {app }, m}=b_{m} F_{\mathrm{ESD}, m}$, to represent the coupling between the ESD actuation force and the mechanical mode in question. $b_{m}$ may be calculated as follows,

$$
b_{m}=\left|\iint_{\mathcal{S}} p_{\mathrm{ESD}}\left(\vec{u}_{m} \cdot \hat{z}\right) \mathrm{d} \mathcal{S}\right|,
$$

where $p_{\mathrm{ESD}}$ is the ESD pressure distribution and $\vec{u}_{m} \cdot \hat{z}$ is the displacement of the mirror's surface along the cavity axis. These quantities have normalisations

$$
\iint_{\mathcal{S}} p_{\mathrm{ESD}} \mathrm{d} \mathcal{S}=1 \text { and } \iiint_{\mathcal{V}} \rho\left|\vec{u}_{m}\right|^{2} \mathrm{~d} \mathcal{V}=1,
$$

$\rho$ being the uniform mass density of the test mass, $\mathcal{S}$ the rear surface of the optic normal to the cavity axis and $\mathcal{V}$ the test mass volume. With this normalisation $\mu_{m}=1$ for all modes.

Thus, including the overlap parameter $b_{m}$ and making use of the proportionality of $Q$ and $R$, i.e.

$$
Q_{\mathrm{eff}, m}=Q_{m} \frac{R_{\mathrm{eff}, m}}{R_{m}},
$$

the magnitude of the required ESD force is given by

$$
F_{\mathrm{ESD}, m}=\sqrt{\mu_{m} k_{B} T} \frac{\omega_{0, m}}{b_{m}}\left(\frac{R_{m}-R_{\mathrm{eff}, m}}{Q_{m} R_{\mathrm{eff}, m}}\right) .
$$




\section{Acknowledgements}

The authors would like to thank Kenneth Strain, Norna Robertson, James Hough and Rainer Weiss for their valuable input to this project. JM's work at The Massachusetts Institute of Technology was carried out under the auspices of the LIGO Visitors Program and supported by the Carnegie Trust for the Universities of Scotland. LIGO was constructed by the California Institute of Technology and the Massachusetts Institute of Technology with funding from the $\mathrm{Na}$ tional Science Foundation and operates under cooperative agreement PHY-0757058. This paper has LIGO Document Number ligo-p1000122.

\section{References}

[1] R. Ward, in: Bulletin of the American Astronomical Society, volume 41, p. 443.

[2] G. Losurdo, Advanced VIRGO webpage, http://wwwcascina.virgo.infn.it/advirgo/, 2010.

[3] K. Kuroda, LCGT Collaboration, Classical and Quantum Gravity 27 (2010) 084004-+.

[4] G. M. Harry, H. Armandula, E. Black, D. R. M. Crooks, G. Cagnoli, J. Hough, P. Murray, S. Reid, S. Rowan, P. Sneddon, M. M. Fejer, R. Route, S. D. Penn, App. Opt. 45 (2006) 1569-1574.

[5] C. M. Caves, Phys. Rev. D 23 (1981) 16931708 .

[6] V. B. Braginsky, S. E. Strigin, S. P. Vyatchanin, Physics Letters A 287 (2001) 331338.

[7] S. E. Strigin, S. P. Vyatchanin, Physics Letters A 365 (2007) 10-16.

[8] M. Evans, L. Barsotti, P. Fritschel, Physics Letters A 374 (2010) 665-671.

[9] S. Gras, C. Zhao, D. G. Blair, L. Ju, Classical and Quantum Gravity 27 (2010) 205019-+.

[10] N. Antler, Passive Damping of a LIGO Mirror, Senior Thesis, Massachusetts Institute of Technology, 2009.

[11] L. Ju, D. G. Blair, C. Zhao, S. Gras, Z. Zhang, P. Barriga, H. Miao, Y. Fan, L. Merrill, Classical and Quantum Gravity 26 (2009) 015002.
[12] J. Degallaix, C. Zhao, L. Ju, D. Blair, Journal of the Optical Society of America B Optical Physics 24 (2007) 1336-1343.

[13] Y. Fan, L. Merrill, C. Zhao, L. Ju, D. Blair, B. Slagmolen, D. Hosken, A. Brooks, P. Veitch, J. Munch, Classical and Quantum Gravity 27 (2010) 084028-+.

[14] S. Gras, D. G. Blair, L. Ju, Physics Letters A 372 (2008) 1348-1356.

[15] F. Acernese et al., Journal of Physics Conference Series 32 (2006) 80-88.

[16] K. Yamamoto, S. Otsuka, M. Ando, K. Kawabe, K. Tsubono, Classical and Quantum Gravity 19 (2002) 1689-1696.

[17] G. Manson, G. Hoffmann de Visme, Journal of Physics D Applied Physics 5 (1972) 13891395.

[18] M. Hewitson, H. Grote, G. Heinzel, K. A. Strain, H. Ward, U. Weiland, Classical and Quantum Gravity 20 (2003) 885.

[19] S. E. Strigin, D. G. Blair, S. Gras, S. P. Vyatchanin, Physics Letters A 372 (2008) 57275731.

[20] COMSOL, http://www.comsol.com/, 2010.

[21] ANSYS, http://www.ansys.com/, 2010.

[22] J. Miller, On Non-Gaussian Beams and Optomechanical Parametric Instabilities in Interferometric Gravitational Wave Detectors, Ph.D. thesis, University of Glasgow, 2010.

[23] A. Cavalleri, G. Ciani, R. Dolesi, M. Hueller, D. Nicolodi, D. Tombolato, S. Vitale, P. J. Wass, W. J. Weber, Physics Letters A 374 (2010) 3365-3369.

[24] R. W. P. Drever, J. L. Hall, F. V. Kowalski, J. Hough, G. M. Ford, A. J. Munley, H. Ward, Applied Physics B: Lasers and Optics 31 (1983) 97-105.

[25] N. A. Robertson, G. Cagnoli, D. R. M. Crooks, E. Elliffe, J. E. Faller, P. Fritschel, S. Goßler, A. Grant, A. Heptonstall, J. Hough, H. Lück, R. Mittleman, M. Perreur-Lloyd, M. V. Plissi, S. Rowan, D. H. Shoemaker, P. H. Sneddon, K. A. Strain, C. I. Torrie, H. Ward, P. Willems, Classical and Quantum Gravity 19 (2002) 4043-4058. 